\title{
Simulation of Ionization and Electronic Excitation in Hypersonic Shock Tube Flows Using DSMC
}

\author{
By Takashi OzawA, ${ }^{1)}$ Satoshi Nomura, ${ }^{1)}$ Taito KawaKami, ${ }^{2)}$ Adrien Lemal, ${ }^{1)}$ and Kazuhisa FuJitA ${ }^{1)}$ \\ ${ }^{1)}$ Research and Development Directorate, JAXA, Chofu, Japan \\ ${ }^{2}$ Shizuoka University, Hamamatsu, Japan
}

(Received June 12th, 2017)

\begin{abstract}
At Japan Aerospace Exploration Agency (JAXA), several sample return missions from deep space have lately been proposed. Since its reentry speed is higher than $10 \mathrm{~km} / \mathrm{s}$, flow conditions are not well known due to highly non-equilibrium phenomena within such speed range. A hyper-velocity shock tube (HVST) at JAXA is capable of producing such flows, and thus, in order to analyze hypersonic flows in HVST, we have developed an unsteady DSMC flow solver and carried out unsteady DSMC flow simulations for a $11.8 \mathrm{~km} / \mathrm{s}$ test case. The computed electron number density was consistent with the estimated value from the VUV measurement in HVST, and the DSMC results show thermally non-equilibrium conditions in the downstream region.
\end{abstract}

Key Words: Shock Tube, Hypersonic Flows, Rarefied Gas Dynamics

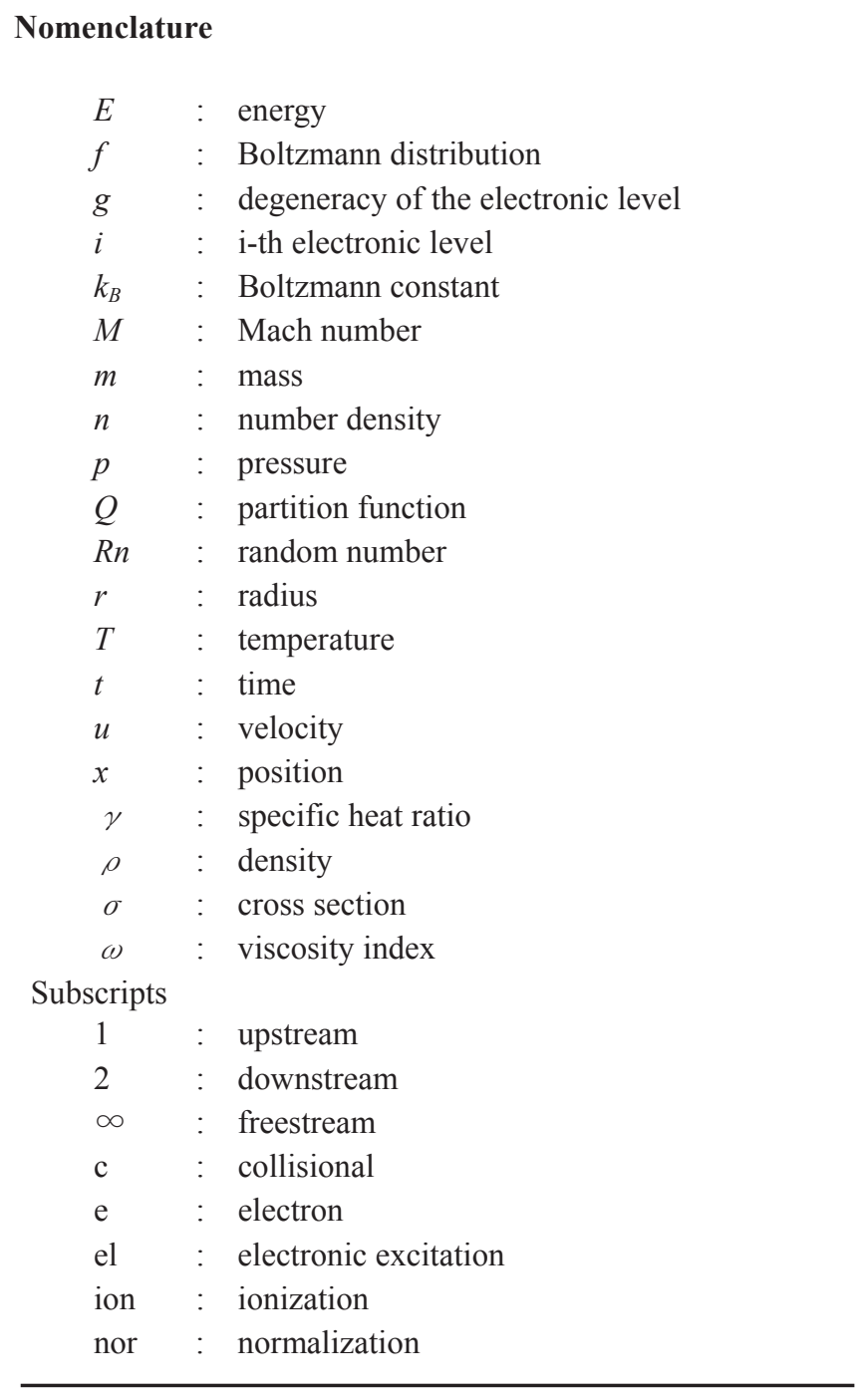

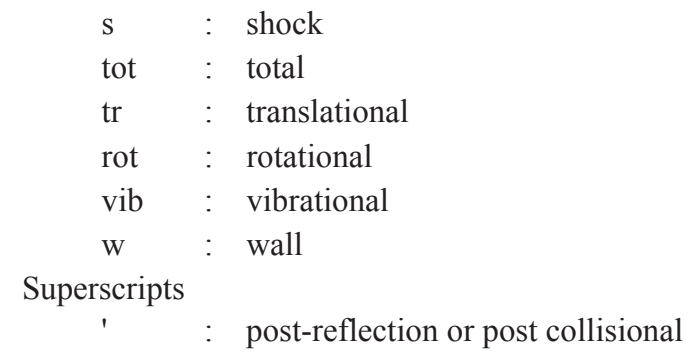

\section{Introduction}

The improvement of aero-thermodynamic prediction accuracy is one of the important factors for the success of atmospheric reentry vehicles, such as Stardust, Hayabusa, and so forth. Lately, sample return missions from deep space, such as Jupiter Trojans, have been proposed at Japan Aerospace Exploration Agency (JAXA). Its estimated reentry speed is faster than that of Hayabusa. Within such speed range, flow conditions are not well known due to highly non-equilibrium phenomena. Therefore, at JAXA a hyper-velocity shock tube $(\text { HVST })^{1,2)}$ has been developed so as to investigate non-equilibrium phenomena in hypersonic flows.

This facility is capable of generating hypersonic flows with a shock speed higher than $10 \mathrm{~km} / \mathrm{s}$. For example, the shock speed is approximately $12 \mathrm{~km} / \mathrm{s}$ at $30 \mathrm{~Pa}$ and $14 \mathrm{~km} / \mathrm{s}$ at $5 \mathrm{~Pa}$ in a low-pressure tube. This facility is equipped with a vacuum-ultraviolet (VUV) measurement system for the analysis of flow conditions in shock region and a triple probe system for the analysis of electron properties. Further details of this facility can be found in Refs. 1) and 2). With regard to HVST, Takayanagi et al. ${ }^{1)}$ have conducted radiation 
measurements in VUV wavelength region behind strong shock wave with a speed between 11 and $14.5 \mathrm{~km} / \mathrm{s}$, and estimated flow conditions in the post-shock region. Lemal et al. ${ }^{3)}$ have analyzed VUV spectra and observed strong non-equilibrium phenomena in the post-shock region. Lately, Nomura et al. ${ }^{4)}$ have measured electron number density distributions by using a triple probe system.

With a shock speed higher than $10 \mathrm{~km} / \mathrm{s}$, a peak translational temperature becomes higher than 40,000 K, where ionization and electronic excitation effects may not be negligible. In addition, computational fluid dynamics (CFD) calculations may not be suitable for low-pressure shock tube flows since insufficient molecular collisions in the shock region result in a high degree of thermal non-equilibrium. Thus, we implement unsteady shock computations using the direct simulation Monte Carlo (DSMC) $)^{5)}$ method in this study to investigate thermally non-equilibrium flow conditions.

Several Groups have studied shock tube flows by using DSMC to date. Strand ${ }^{6}$ has implemented unsteady onedimensional (1D) DSMC simulations for $8 \mathrm{~km} / \mathrm{s}$ shock flows with the moving-window sampling method. $\mathrm{Li}^{7}$ and $\mathrm{Zhu}$ et $a l^{8,9)}$ have also carried out unsteady DSMC flow simulations for shock flows up to $9 \mathrm{~km} / \mathrm{s}$.

In this work, we first develop an unsteady DSMC flow solver integrating with ionization and electronic excitation models. Second, we carry out unsteady DSMC flow simulations for HVST test cases, and investigate ionization and electronic excitation effects in unsteady shock flows. Finally, we compare computed results with measured ones, and analyze non-equilibrium phenomena.

\section{Numerical Modeling}

In order to capture non-equilibrium phenomena, we first extend a DSMC code, named Modeling Of Transitional-Ionized Flows (MOTIF), which has been developed at JAXA to simulate rarefied nozzle flows or planetary atmospheric flows, to unsteady flow solver. In this work, we apply the piston method ${ }^{5)}$ to $1 \mathrm{D}$ unsteady shock tube flows. In this method, we need the following input parameters: piston velocity $u_{\mathrm{w}}$, upstream pressure $p_{1}$, and upstream temperature $T_{1}$. The left boundary works as a moving piston with the velocity $u_{\mathrm{w}}$. The cell boundaries and volumes become a function of time and must be recalculated at each time step. The post-reflection velocity component of the molecule is

$$
u^{\prime}=2 u_{w}-u .
$$

The post reflection location of the particle, $x^{\prime}$, is

$$
x^{\prime}=2 x_{w}-x+u^{\prime} d t .
$$

Any other particle properties remain unchanged during the reflection procedure.

The definition of shock position is obtained as follows. The normalized pressure is

$$
p_{\text {nor }}=\frac{p-p_{1}}{p_{2}-p_{1}}
$$

Table 1. A shock flow condition in HVST.

\begin{tabular}{ll}
\hline Definition & Value \\
\hline Initial pressure in the low-pressure tube & $30 \mathrm{~Pa}$ \\
Initial temperature. & $300 \mathrm{~K}$ \\
Shock velocity & $11.77 \mathrm{~km} / \mathrm{s}$ \\
Pressure behind a shock & $45.043 \mathrm{kPa}$ \\
Gas velocity behind a shock & $11.04 \mathrm{~km} / \mathrm{s}$ \\
Density behind a shock & $5.56 \times 10^{-3} \mathrm{~kg} / \mathrm{m}^{3}$ \\
\hline
\end{tabular}

The shock position is defined as the position where the normalized pressure is equal to 0.5 .

To simulate air shock tube flows in HVST, eleven chemical species $\left(\mathrm{N}, \mathrm{O}, \mathrm{N}^{+}, \mathrm{O}^{+}, \mathrm{N}_{2}, \mathrm{O}_{2}, \mathrm{NO}, \mathrm{N}_{2}^{+}, \mathrm{O}_{2}{ }^{+}, \mathrm{NO}^{+}\right.$and $\left.\mathrm{e}^{-}\right)$are considered. The nominal HVST test condition is shown in Table 1. The initial chemical composition is $80 \% \mathrm{~N}_{2}$ and $20 \%$ $\mathrm{O}_{2}$.

For most reentry conditions, dissociation reactions and ionization reactions play an important role. In this work, we consider 55 chemical reactions, and the total collision energy (TCE) model $^{6)}$ is utilized for both charged and neutral species. Details of the chemical reaction dataset and parameters for the modified Arrhenius form,

$$
K_{f}=A T^{n} \exp \left(-E_{a} / k T\right)
$$

can be found in Ref 10).

For modeling the molecular collision frequency, the no time counter (NTC) scheme ${ }^{5)}$ is employed, and the variable hard sphere (VHS) model is used for modeling the collision cross section between particles if it is not specified individually. For modeling rotation-translation (R-T) and vibration- translation (V-T) energy transfers between neutral species, the Borgnakke-Larsen (BL) model ${ }^{11)}$ with temperature- dependent rotational and vibrational relaxation numbers is used. Also, a correction term proposed by Park to the V-T relaxation time is employed for enhancement of coupling between vibrational and rotational modes.

Similar to DSMC1U, an ensemble average scheme has been used in this work, and the average iteration is approximately 100. Note that the time step, cell size, computational domain, and the total number of simulated particles have been investigated to obtain results that are independent of these DSMC numerical parameters. The time step for the HVST cases is generally $5 \mathrm{~ns}$. The cell size is set to be smaller than the upstream local mean free path, and the average number of simulated molecules is roughly a few million. Behind the shock, the average number of simulated particles per cell is a

Table 2. Ionization reactions for air shock tube conditions.

\begin{tabular}{ccccc}
\hline Reaction & $A, \mathrm{~m}^{3} / \mathrm{s}$ & $n$ & $E_{\mathrm{a}}, \mathrm{J}$ & $T$ \\
\hline $\begin{array}{c}(1) \mathrm{N}+\mathrm{O} \rightarrow \\
\mathrm{NO}^{+}+\mathrm{e}^{-}\end{array}$ & $8.80 \times 10^{-18}$ & 0.0 & $4.40 \times 10^{-19}$ & $T_{\text {tr }}$ \\
$(2) \mathrm{N}+\mathrm{N} \rightarrow$ & $3.39 \times 10^{-17}$ & 0.0 & $9.32 \times 10^{-19}$ & $T_{\text {tr }}$ \\
$\mathrm{N}_{2}^{+}+\mathrm{e}^{-}$ & & & & \\
$(3) \mathrm{O}+\mathrm{O} \rightarrow$ & $1.83 \times 10^{-17}$ & 0.0 & $1.11 \times 10^{-18}$ & $T_{\text {tr }}$ \\
$\mathrm{O}_{2}^{+}+\mathrm{e}^{-}$ & & & & \\
$(4) \mathrm{e}^{-}+\mathrm{N} \rightarrow$ & $9.0 \times 10^{-11}$ & -1.0 & $1.50 \times 10^{-18}$ & $T_{\mathrm{e}}$ \\
$\mathrm{N}^{+}+2 \mathrm{e}^{-}$ & & & & \\
$(5) \mathrm{e}^{-}+\mathrm{O}^{-} \rightarrow$ & $3.0 \times 10^{-10}$ & -1.0 & $1.40 \times 10^{-18}$ & $T_{\mathrm{e}}$ \\
$\mathrm{O}^{+}+2 \mathrm{e}^{-}$ & & & & \\
\hline
\end{tabular}


few thousands, and for instance, the number of samples for atomic species in each cell per time step is greater than 10,000 in the downstream region. Thus, if electronic excitation population at an electronic level is higher than $0.1 \%$ in the region, the electronic excitation distributions can be reliable for the level.

\subsection{Modeling of ionization in DSMC}

In order to simulate air shock tube flows with a speed higher than $10 \mathrm{~km} / \mathrm{s}$, important ionization reactions are listed in Table 2. Atomic $\mathrm{N}$ and $\mathrm{O}$ are produced by dissociation processes, and their collisions result in ionization processes. Reactions (1) through (3) are treated as exchange reactions and reactions (4) and (5) are treated as electron impact (EI) ionization reactions.

For collision processes between neutral particles, we use general VHS cross sections. On the other hand, for electron-neutral (e-n) collisions, we employ the modified VHS model in Ref. 12) in order to represent resonance peaks. For electron-ion collisions, the Coulomb collision model ${ }^{13)}$ is utilized instead of the VHS model. For the movement of charged species, a single time step, exact charge neutrality $(\mathrm{ECN})^{12)}$ method is used.

In addition, electron-vibrational (e-V) energy transfer for $\mathrm{N}_{2}$ plays an important role in air ionized hypersonic flows. Lee ${ }^{14)}$ developed an expression for the e-V relaxation time for $\mathrm{N}_{2}$ using a master equation approach. He found that the maximum value of the rate coefficient for each vibrational state lies around $16,000 \mathrm{~K}$ and the minimum relaxation time lies around 7,000 $\mathrm{K}$ due to the strong shape resonance effect. In this work, the e- $\mathrm{V}$ relaxation time with the resonance effect correction is employed.

\subsection{Modeling of electronic transitions in DSMC}

Since the electronic energy and the EI ionization rates of atomic species $\mathrm{N}$ and $\mathrm{O}$ were found to be important from our previous work, ${ }^{15)}$ to determine hypersonic flow characteristics with its speed higher than $10 \mathrm{~km} / \mathrm{s}$, the discrete electronic energy mode is added for atomic species. Although several electronic transition databases ${ }^{16)}$ are available, we employ a 22 electronic-level Park model ${ }^{17)}$ for atomic nitrogen and 19 electronic-level Park model ${ }^{17)}$ for atomic oxygen in this work from the practical view point. Note that for polyatomic species, only three (translational, rotational and vibrational) energy modes are considered, and electronic energies for these species are not taken into account yet since the addition of electronic modes for polyatomic species does not impact shock flow properties significantly compared to atomic ones.

Recently, the Quantum-Kinetic (Q-K) chemical reaction model $^{18-20)}$ that predict equilibrium and nonequilibrium reaction rates using only kinetic theory and fundamental molecular properties has been developed. The essential advantage of the Q-K model is that no macroscopic information is necessary in the model. In this work, an accumulated-distribution (A-D) scheme ${ }^{12)}$ has also been employed to improve the efficiency of the Q-K model for electronic energy transitions.

A post-collisional energy redistribution mechanism that involves electronic transitions only depends on the collisional energy of a pair. Similar to R-T and V-T energy transfers, the
BL model is utilized for post-collisional energy redistribution for electron-electronic (e- $\left.E_{\mathrm{el}}\right)$ and electronic-translational $\left(E_{\mathrm{el}}-E_{\mathrm{tr}}\right)$ energy transfers. The joint distribution between translational and electronic modes for sampling a post-collision electronic energy level $i^{\prime}$ from the collisional energy $\left(E_{\mathrm{c}}=E_{\mathrm{tr}}+\mathrm{E}_{\mathrm{el}}^{i}=E_{\mathrm{tr}}^{\prime}+E_{\mathrm{el}}^{i^{\prime}}\right)$ can be written as

$$
f\left(i^{\prime} ; E_{c}\right)=\frac{\exp \left(-E_{c} / k_{B} T\right)}{\Gamma(5 / 2-\omega)} \frac{g_{i^{\prime}}\left[\left(E_{c}-E_{e l}^{i^{\prime}}\right) / k_{B} T\right]^{3 / 2-\omega}}{Q_{e l}} .
$$

This equation can be simplified as

$$
f\left(i^{\prime} ; E_{c}\right) \propto g_{i^{\prime}}\left(E_{c}-E_{e l}^{i^{\prime}}\right)^{3 / 2-\omega}
$$

The accumulated distribution is

$$
F\left(i^{\prime} ; E_{c}\right)=\sum_{j=1}^{i^{\prime}} g_{j}\left(E_{c}-E_{e l}^{j}\right)^{3 / 2-\omega}, \quad F_{n o r}\left(i^{\prime} ; E_{c}\right)=\frac{F\left(i^{\prime} ; E_{c}\right)}{F\left(I_{c, \max } ; E_{c}\right)} .
$$

The procedure of the A-D method is as follows:

(1) calculate the total collisional energy, $E_{\mathrm{c}}$, of a pair of particles and determine the maximum allowable electronic energy level, $i_{\mathrm{c}, \max }$,

(2) select energetically possible transitions using the trial BL redistribution,

(3) calculate the normalized accumulated distribution $F_{\text {nor }}$ $\left(i^{\prime} ; E_{\mathrm{c}}\right)$ for possible transitions,

(4) find the value of $i^{\prime}$ for which $F_{\text {nor }}\left(i^{\prime}-1 ; E_{\mathrm{c}}\right) \leqq R n<F_{\text {nor }}\left(i^{\prime} ; E_{\mathrm{c}}\right)$ using the bisection method, where $R n$ is a uniformly

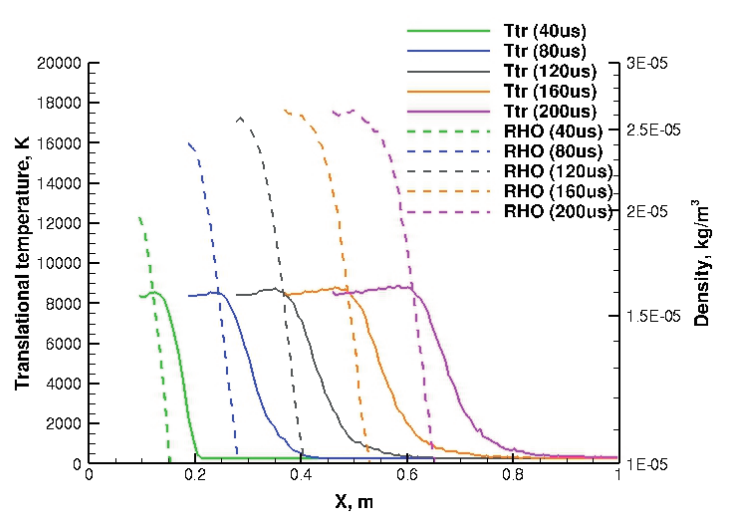

Fig. 1. Change in temperature and density in Ar shock flows $\left(M_{\mathrm{s}}=10\right)$.

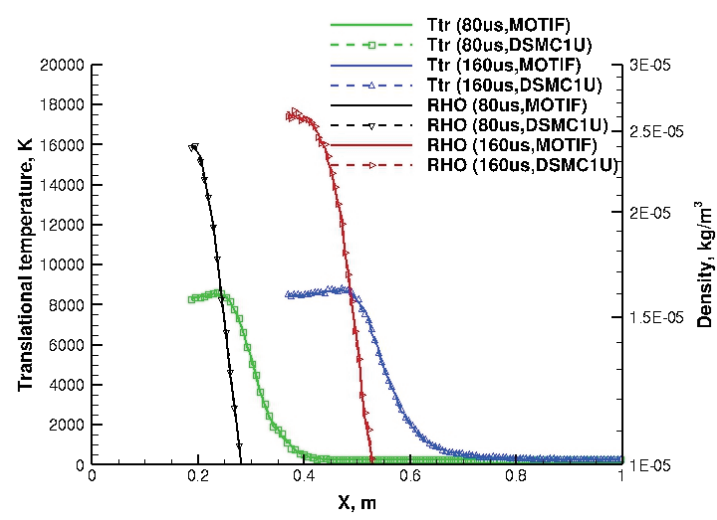

Fig. 2. Comparison of temperature and density for Ar shock flows $\left(M_{\mathrm{s}}=10\right)$ between DSMC1U and MOTIF. 
distributed random number between 0 and 1 .

\section{Results and Discussions}

\subsection{Test case $1\left(\mathrm{Ar}, M_{\mathrm{s}}=10\right)$}

First, we have carried out 1D unsteady DSMC calculations for a test case as the validation of our unsteady code. The test condition is as follows. The piston velocity $\left(u_{\mathrm{w}}\right)$ is $2.285 \mathrm{~km} / \mathrm{s}$, and the upstream pressure $\left(p_{1}\right)$ and temperature $\left(T_{1}\right)$ are 0.38 $\mathrm{Pa}$ and $273 \mathrm{~K}$, respectively. The chemical composition is $100 \%$ argon, and the upstream mean free path is approximately $1.3 \times 10^{-2} \mathrm{~m}$. Argon unsteady shock flows were computed by two codes, DSMC1U of Bird ${ }^{5)}$ and MOTIF. Figure 1 shows change in temperature and density in the argon shock flows. The downstream temperature gradually increases within $120 \mu \mathrm{s}$, and both density and temperature become uniform after $120 \mu \mathrm{s}$. The shock velocity is approximately 3.1 $\mathrm{km} / \mathrm{s}$, and the corresponding Mach number is roughly 10. In Fig. 2, our results obtained by the MOTIF code are compared with those of DSMC1U. The density and temperature are consistent with each other at both developing phase $(80 \mu \mathrm{s})$ and developed phase $(160 \mu \mathrm{s})$, and thus, the MOTIF results are validated for this test case.

\subsection{Test case 2 (Air, $5 \mathrm{~km} / \mathrm{s}$ )}

Second, we have carried out a test case to investigate the impact of chemical reactions. The test condition is as follows. The piston velocity $\left(u_{\mathrm{w}}\right)$ is $5.0 \mathrm{~km} / \mathrm{s}$, and the upstream pressure $\left(p_{1}\right)$ and temperature $\left(T_{1}\right)$ are $30 \mathrm{~Pa}$ and $273 \mathrm{~K}$, respectively. The chemical composition is $80 \% \mathrm{~N}_{2}$ and $20 \% \mathrm{O}_{2}$, and the
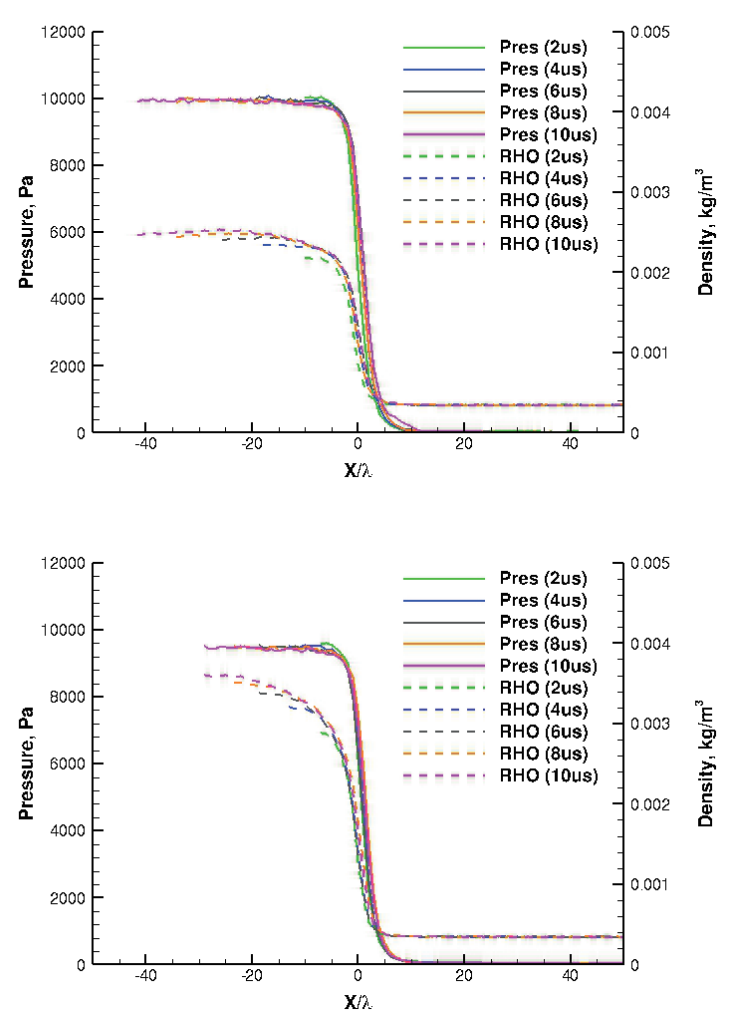

Fig. 3. Comparison of pressure and density distributions between cases without (top) and with (bottom) chemical reactions at $u_{2}=5 \mathrm{~km} / \mathrm{s}$. upstream mean free path is approximately $2.0 \times 10^{-4} \mathrm{~m}$.

Figure 3 shows a comparison of pressure and density distributions between two cases with and without chemical reactions between 2 and $10 \mu \mathrm{s}$. Note that the $x$ axis is normalized by the upstream mean free path. For both cases, the shock structure is converged at $10 \mu$ s within the limit of 20 $\lambda$ from the shock front, and the downstream pressure is approximately $1.0 \times 10^{4} \mathrm{~Pa}$. Figure 4 presents a comparison of temperature distributions between two cases with and without chemical reactions. Without chemical reactions, the downstream temperature is approximately $14000 \mathrm{~K}$, and the rotational temperature is equilibrated within $2 \mathrm{~mm}$ from the temperature overshoot position. The vibrational relaxation is much slower, and needs nearly $8 \mathrm{~mm}(40 \lambda)$ for equilibration. For this case, the shock velocity and the corresponding Mach number is roughly $5.8 \mathrm{~km} / \mathrm{s}$ and 17.5 , respectively. On the
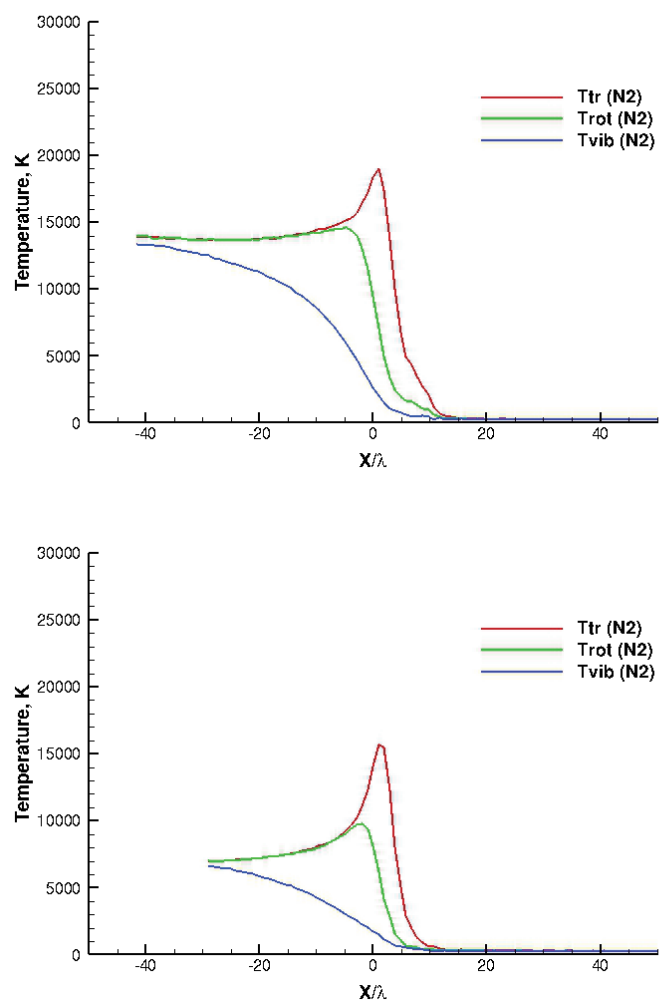

Fig. 4. Comparison of temperature distributions between cases without (top) and with (bottom) chemical reactions at $u_{2}=5 \mathrm{~km} / \mathrm{s}$.

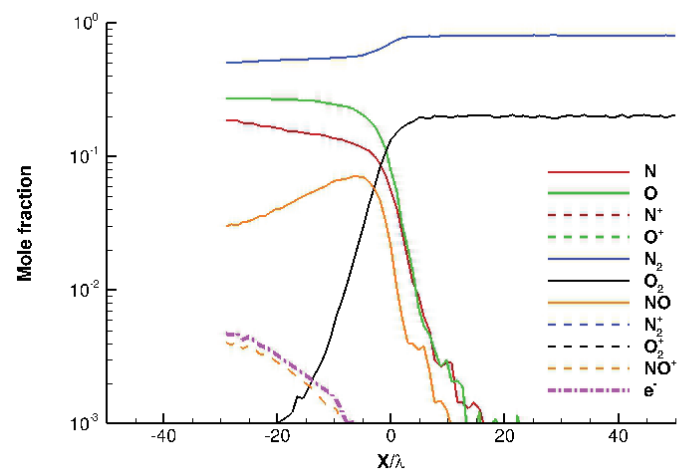

Fig. 5. Comparison of mole fraction distributions at $u_{2}=5 \mathrm{~km} / \mathrm{s}$. 
other hand, with chemical reactions, the downstream temperature is considerably decreased due to energy consumption, and approximately $6000 \mathrm{~K}$. With respect to temperature, the equilibrium region is about $35 \lambda$ downstream from the shock front. The energy consumption in the downstream region results in the decrease of the shock speed, which is approximately $5.5 \mathrm{~km} / \mathrm{s}\left(M_{\mathrm{s}}=16.5\right)$. Figure 5 shows the mole fraction distribution for the case with chemical and ionization reactions. In the downstream region, while $\mathrm{NO}^{+}$is produced from $\mathrm{N}$ and $\mathrm{O}$ collisions, the degree of ionization is basically lower than $1 \%$, and thus, the ionization reactions do not impact the flow field. $\mathrm{N}, \mathrm{O}$, and $\mathrm{NO}$ rapidly increase near the shock front, and $\mathrm{O}_{2}$ is fully dissociated within $20 \lambda(4 \mathrm{~mm})$ from the shock front. In the downstream region, although NO gradually decreases along the downstream direction, $\mathrm{NO}^{+}$and $\mathrm{N}$ slightly increase.

\subsection{HVST shock tube flows $\left(u_{\mathrm{s}}=11.8 \mathrm{~km} / \mathrm{s}\right)$}

As a nominal HVST test case, we have carried out 1D unsteady DSMC computations at a shock velocity of 11.8 $\mathrm{km} / \mathrm{s}$. The piston velocity $\left(u_{\mathrm{w}}\right)$ is $11.0 \mathrm{~km} / \mathrm{s}$, and the upstream pressure $\left(p_{1}\right)$ and temperature $\left(T_{1}\right)$, are $30 \mathrm{~Pa}$ and $300 \mathrm{~K}$ respectively. The chemical composition is $80 \% \mathrm{~N}_{2}$ and $20 \%$ $\mathrm{O}_{2}$, and the upstream mean free path is approximately $2.0 \times$ $10^{-4} \mathrm{~m}$.

In this study, we focus on the shock structure development and the flow conditions in downstream region near the shock front. First, we investigate the impact of ionization reactions on the flow field for this 11.8 shock velocity case. Note that electronic transitions are not activated yet. In Fig. 6, we compare pressure and density distributions between two cases with and without ionization reactions between 2 and $10 \mu$ s. Similarly, Figure 7 shows a comparison of temperature distributions between the two cases. It can be seen in the figures that two results at $8 \mu \mathrm{s}$ and $10 \mu \mathrm{s}$ are identical, and thus, the shock structure for both cases is basically converged at 10 $\mu \mathrm{s}$ within the limit of $20 \lambda$ from the shock front. The downstream pressure is approximately $5.0 \times 10^{4} \mathrm{~Pa}$ without ionization reactions and $4.3 \times 10^{4} \mathrm{~Pa}$ with ionization reactions. In the downstream region, the density keeps increasing toward the downstream due to ionization reactions. Correspondingly, temperatures keep decreasing toward the downstream with ionization reactions. Without ionization reactions, the translational temperature at the overshoot and in the downstream region is approximately $70,000 \mathrm{~K}$ and $36,000 \mathrm{~K}$, respectively. Since particle energies are not consumed by ionization reactions, the shock velocity is about $1 \mathrm{~km} / \mathrm{s}$ faster than that of the case with ionization reactions. On the other hand, for the case with ionization reactions, the change in density and temperature in the downstream region is mainly due to ionization reactions, especially electron impact ionization reactions. The downstream temperature is significantly decreased with ionization reactions due to energy consumption, and the translational temperature at the overshoot and in the downstream region (20 $\lambda$ from the shock front) is approximately $50,000 \mathrm{~K}$ and $10,000 \mathrm{~K}$, respectively. Thus, for this velocity range, the impact of ionization reactions cannot be negligible.

Second, we investigate the impact of electronic excitation transitions on the flow field for the shock velocity of 11.8 $\mathrm{km} / \mathrm{s}$ case by activating electronic transitions for $\mathrm{N}$ and $\mathrm{O}$.
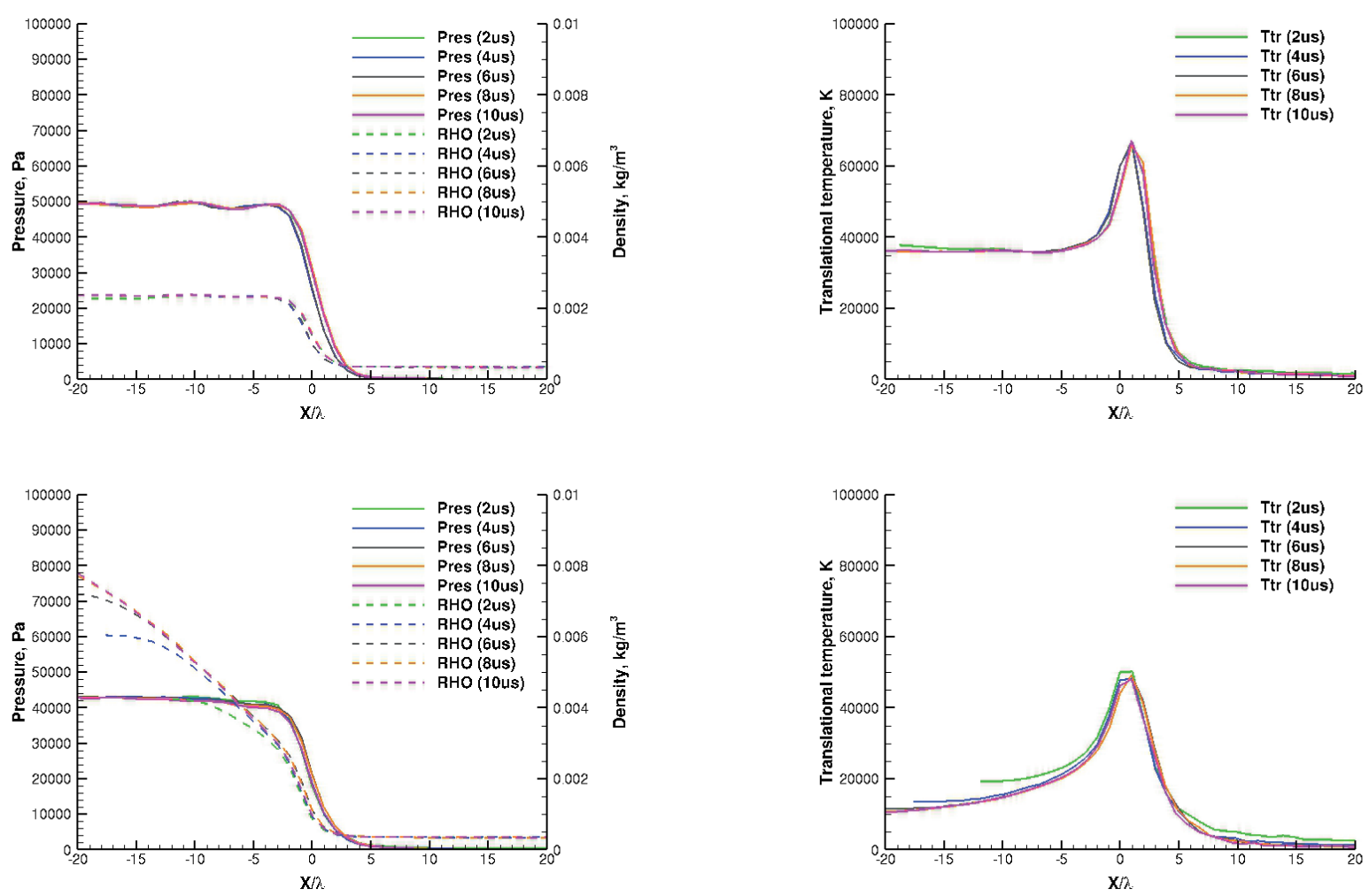

Fig. 6. Comparison of pressure and density distributions between cases without (top) and with (bottom) ionization reactions at $u_{\mathrm{s}}=11.8 \mathrm{~km} / \mathrm{s}$.

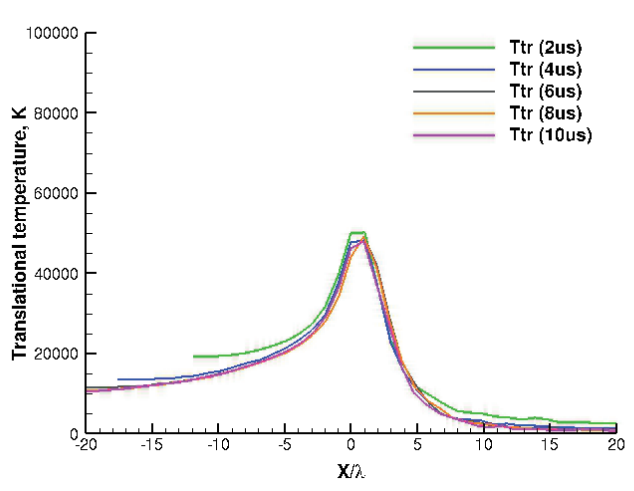

Fig. 7. Comparison of temperature distributions between cases without (top) and with (bottom) ionization reactions at $u_{\mathrm{s}}=11.8 \mathrm{~km} / \mathrm{s}$. 
Since electronically excited particles can promote ionization reactions, the degree of ionization is increased by $5 \%$ with electronic transitions at $20 \lambda$ downstream from the shock front (see Fig. 8). Figure 9 shows a comparison of temperature distributions between cases with and without electronic excitation transitions. Without electronic transitions, four temperatures (translational, rotational, vibrational, and electron) are equilibrated within roughly $10 \lambda$ from the temperature overshoot position, and the equilibrium temperature at $20 \lambda$ from the shock front is approximately $10,500 \mathrm{~K}$. With electronic transitions, translational, rotational, and vibrational temperatures are slightly decreased whereas free electron temperature is lowered by roughly $1,000 \mathrm{~K}$. Electronic temperature, $T_{\mathrm{el}}$, is computed in accordance with the population ratio between the ground level and the first excited energy level. The electronic temperature is approximately $6,200 \mathrm{~K}$, and the lower electronic temperature compared to the equilibrium temperature is consistent with the phenomena in Ref. 3). Lemal et al. ${ }^{3)}$ estimated electronic temperature from VUV emission spectra to be $20 \%$ lower than the equilibrium temperature. This feature is attributed to the strong depletion of the electronic states by electron impact processes.

In Fig. 10, we compare electronic excitation populations of $\mathrm{N}$ and $\mathrm{O}$ in the downstream region between DSMC and the Boltzmann distribution at electronic temperature. In DSMC, only three energy levels for $\mathrm{N}$ can be seen in the figure, and the DSMC result basically agrees with the Boltzmann distribution at $T_{\mathrm{el}}$. For O, DSMC predicts higher populations at the first and second excited energy levels than those of the Boltzmann distribution. This is attributed to the lower electron impact ionization rate for $\mathrm{O}$ in the downstream region compared to $\mathrm{N}$.

In Fig. 11, we compare temperature distributions between DSMC and CFD in Ref. 3). Note that the shock velocity is $11.8 \mathrm{~km} / \mathrm{s}$ in DSMC and $12.5 \mathrm{~km} / \mathrm{s}$ in CFD. In CFD, a two-temperature model has been used, and the details can be found in Ref. 3). While translational temperature distributions are consistent with each other, DSMC predicts higher vibrational temperature behind the overshoot region. This originates from the discrepancy of temperature models between DSMC and CFD. Although a five-temperature model has been used in DSMC, a two-temperature model has been used in CFD. In DSMC, the slower relaxation from vibrational mode results in higher vibrational temperature than that of CFD. In the downstream region, the discrepancy in vibrational temperature decreases. As mentioned earlier, electron and electronic temperatures in DSMC are lower than the other temperatures.

We also compare electron number density distributions among DSMC, CFD and measured data (Ref. 3) in Fig. 12. In Ref. 3), the measured electron number densities were inferred from the broadening of hydrogen emission lines of alpha and beta, and these data are also shown in the figure. DSMC predicts slightly higher electron number density in the downstream region than CFD. It is notable that in DSMC termolecular collisions are not considered, and thus, ionization rates can be overestimated in the farther downstream region.
Because of the temperature model difference between DSMC and CFD, charged particles are produced in farther upstream region in CFD. On the other hand, the DSMC result shows higher diffusion of charged species toward the upstream. In DSMC, light particles diffuse farther in non-continuum upstream region compared to continuum diffusion models.

Finally, we discuss the uncertainty of shock velocity in the HVST measurement. Although the shock velocity determination is crucial in HVST, its dependence on measurement methods is not well-known. Thus, in this work, we discuss the difference between pressure sensor and laser Schlieren methods since these two methods are utilized for HVST shock velocity measurement. While the pressure sensor
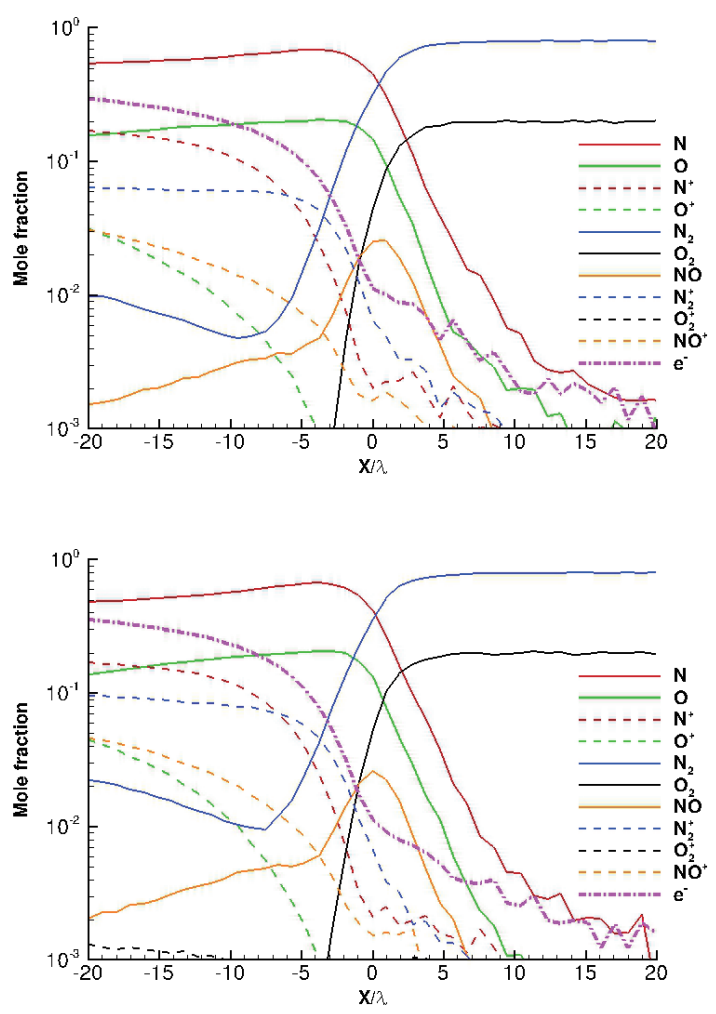

Fig. 8. Comparison of mole fraction distributions at $u_{\mathrm{s}}=11.8 \mathrm{~km} / \mathrm{s}$ between cases without (top) and with (bottom) electronic excitation transitions.

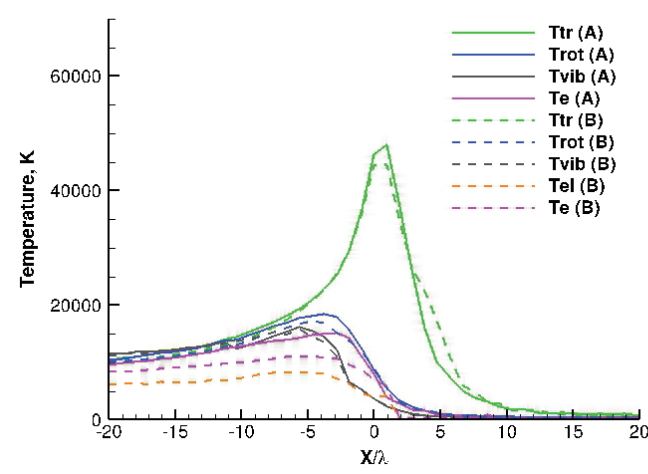

Fig. 9. Comparison of temperature distributions at $u_{\mathrm{s}}=11.8 \mathrm{~km} / \mathrm{s}$ between cases without electronic transitions (A) and with electronic transitions (B). 


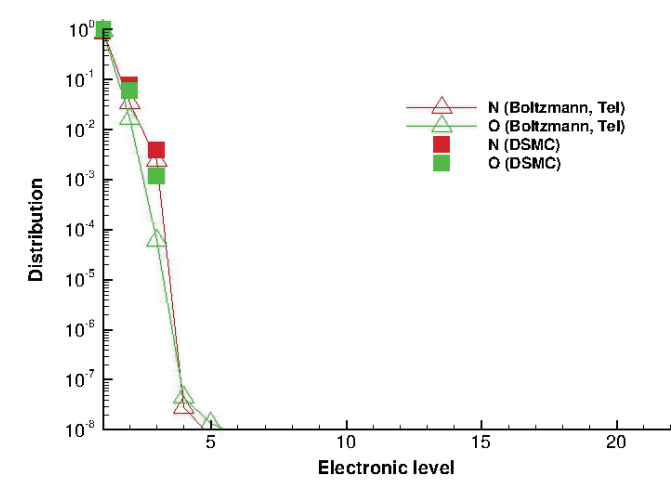

Fig. 10. Comparison of electronic excitation populations of $\mathrm{N}$ and $\mathrm{O}$ in the downstream region between DSMC and the Boltzmann distribution at electronic temperature.

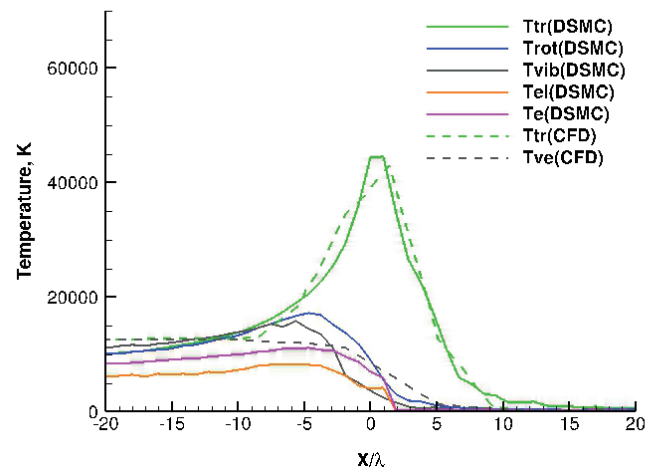

Fig. 11. Comparison of temperature distributions between DSMC $\left(u_{\mathrm{s}}=11.8 \mathrm{~km} / \mathrm{s}\right)$ and CFD $\left(u_{\mathrm{s}}=12.5 \mathrm{~km} / \mathrm{s}\right)$ in Ref. 3 .

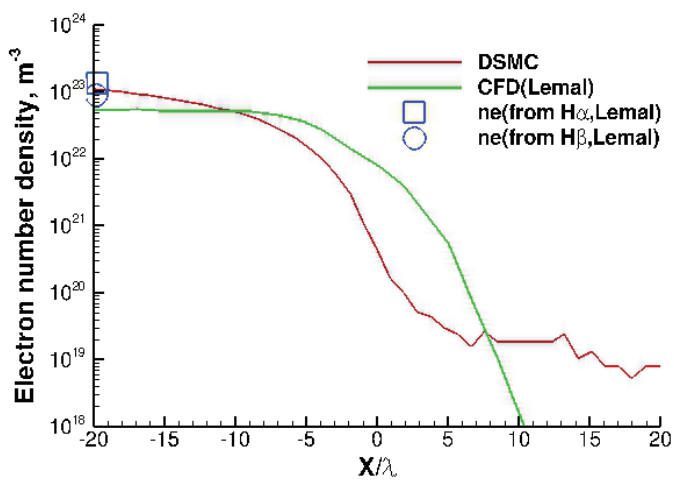

Fig. 12. Comparison of electron number density distributions between $\operatorname{DSMC}\left(u_{\mathrm{s}}=11.8 \mathrm{~km} / \mathrm{s}\right)$ and CFD $\left(u_{\mathrm{s}}=12.5 \mathrm{~km} / \mathrm{s}\right)$ in Ref. 3$)$.

detects the pressure increase of the shock front, the laser detects the density increase. Therefore, the discrepancy between pressure and density distributions in the shock front should be analyzed for the HVST shock velocity measurement. The pressure threshold was estimated to be $1 \mathrm{kPa}$, and the density threshold was set to $0.003 \mathrm{~kg} / \mathrm{m}^{3}$ in this work. As shown in Fig. 6, the discrepancy of shock front detection between pressure and density increase is approximately 1.0 $\mathrm{mm}$ for the $11.8 \mathrm{~km} / \mathrm{s}$ case. Since the spatial resolution of the shock velocity measurement system is estimated to be $2 \mathrm{~mm}$, and thus, the discrepancy may not affect the shock velocity data for this case.

\section{Conclusions}

At JAXA, several sample return missions from deep space have lately been proposed, and the study of aerothermodynamic characteristics for hypersonic flows with a speed higher than $10 \mathrm{~km} / \mathrm{s}$ is in high demand. HVST is capable of producing such flows, and thus, in order to analyze hypersonic flows in HVST, we have developed an unsteady DSMC flow solver integrating with ionization and electronic excitation models. We have carried out unsteady DSMC flow simulations for a HVST test case with $u_{\mathrm{s}}$ of $11.8 \mathrm{~km} / \mathrm{s}$. Good agreement in translational temperature was obtained between DSMC and CFD in Ref. 3), and in the downstream region, the computed electron number density was consistent with the estimated value from the VUV measurement. We computed five temperatures (translational, rotational, vibrational, electronic, and electron) in DSMC, and found thermally nonequilibrium conditions in the downstream region.

For our future work, we will first conduct unsteady DSMC computations at a shock velocity of $14.5 \mathrm{~km} / \mathrm{s}$ at $5 \mathrm{~Pa}$ and analyze low-pressure shock tube conditions. Second, we will extend the computational time and geometry for the analysis of further downstream flow conditions. Third, radiation effect will be coupled with DSMC computations.

\section{References}

1) Takayanagi, H., Fujita, K., Ishida, H., Yamada, K., and Abe, T.: Radiation Intensity Measurement in VUV Wavelength Region behind Strong Shock Wave for Future Sample Return Missions, AIAA Paper 2014-2965, 2014.

2) Nishimura, S., Takayanagi, H., Nomura, S., Fujita, K., and Matsui, M.: Speeding up of Shock Wave for Future Missions and Spectroscopic Measurement of Strong Shock Wave, 2015-e-32, 30th International Symposium on Space Technology and Science, Kobe, Hyogo, July 4-10, 2015.

3) Lemal, A., Nishimura, S., Nomura, S., Takayanagi, H., Matsuyama, S., and Fujita, K.: Analysis of VUV Radiation Measurements from High Temperature Air Mixtures, AIAA Paper 2016-0740, 2016.

4) Nomura, S., Lemal, A., and Fujita, K.: Precursor Electron Measurements with Electrostatic Probes and Spectroscopy, The 31st International Symposium on Shock Waves, Nagoya, Japan, July 9-14, 2017.

5) Bird, G. A.: Molecular Gas Dynamics and the Direct Simulation of Gas Flows, Clarendon, Oxford, England, U.K., 1994.

6) Strand, J. S.: Statistical Methods for the Analysis of DSMC Simulation of Hypersonic Shocks, Ph.D. thesis, The University of Texas at Austin, 2012.

7) Li, Z., Zhu, T., and Levin, D. A.: DSMC Simulation of Vibrational Excitation and Reaction for Molecular Nitrogen in Shock Tube Flows. AIAA Paper 2013-1201, 2013.

8) Zhu, T., Li, Z., and Levin, D. A.: Modeling of NO Radiation from Unsteady and Steady Shocks Using DSMC. AIAA Paper 2013-2786, 2013.

9) Zhu, T., Li, Z., and Levin, D. A.: Simulation of Radiation Generated by Chemical Reactions in Weakly Ionized Shock Waves Using DSMC, AIAA Paper 2014-1212, 2014.

10) Ozawa, T., Zhong, J., and Levin, D. A.: Development of Kinetic-based Energy Exchange Models for Noncontinuum, Ionized Hypersonic Flows, Physics of Fluids, 20 (2008), 046102.

11) Borgnakke, C. and Larsen, P. S.: Statistical Collision Model for Monte Carlo Simulation of Polyatomic Gas Mixture, Journal of Computational Physics, 18 (1975), pp. 405-420.

12) Ozawa, T., Suzuki, T., Takayanagi, H. and Fujita, K.: Analysis of Non-Continuum Hypersonic Flows for the Hayabusa Reentry, AIAA 
Trans. JSASS Aerospace Tech. Japan Vol. 16, No. 2 (2018)

Paper 2011-3311, 2011.

13) Kim, M., Gulhan, A., and Boyd, I. D.: Modeling of Electron Temperature in Hypersonic Flows, AIAA paper 2011-1028, 2011.

14) Lee, J. H.: Electron-Impact Vibrational Relaxation in HighTemperature Nitrogen, Journal of Thermophysics and Heat Transfer, 7 (1993), pp. 399-405.

15) Li, Z., Ozawa, T., Sohn, I. and Levin, D. A.: Modeling of Electronic Excitation and Radiation in Non-continuum Hypersonic Reentry Flows, Physics of Fluids, 23 (2011), 066102.

16) Frost, R. M. and Awakowicz, N. R.: Calculated Cross Sections and Measured Rate Coefficients for Electron-Impact Excitation of Neutral and Singly Ionized Nitrogen, Journal of Applied Physics, $\mathbf{8 4}$ (1998), pp. 2989-3003.
17) Park, C.: Nonequilibrium Hypersonic Aerothermodynamics, John Wiley\&Sons, Inc., New York, 1990.

18) Bird, G. A.: A Comparison of Collision Energy-based and Temperature-based Procedures in DSMC, Rarefied Gas Dynamics: 26th International Symposium, Kyoto, Japan, 20-25 July 2008, edited by T. Abe, 1084 (2009), pp. 245-250.

19) Gallis, M. A., Bond, R. B., and Torczynski, J. R.: A Kinetic-Theory Approach for Computing Chemical-Reaction Rates in Upper-Atmosphere Hypersonic Flows, The Journal of Chemical Physics, 131 (2009), 124311.

20) Liechty, D. S. and Lewis, M.: Treatment of Electronic Energy Level Transition and Ionization Following the Particle- Based Chemistry Model, AIAA paper 2010-449, 2010. 